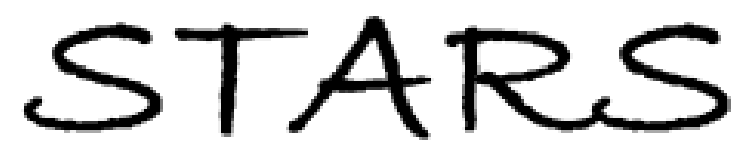

University of Central Florida

STARS

$1-1-2011$

\title{
Photoassociation of a cold-atom-molecule pair. II. Second-order perturbation approach
}

\author{
M. Lepers \\ R. Vexiau \\ N. Bouloufa \\ O. Dulieu \\ Viatcheslav Kokoouline \\ University of Central Florida
}

Find similar works at: https://stars.library.ucf.edu/facultybib2010 University of Central Florida Libraries http://library.ucf.edu

This Article is brought to you for free and open access by the Faculty Bibliography at STARS. It has been accepted for inclusion in Faculty Bibliography 2010 s by an authorized administrator of STARS. For more information, please contact STARS@ucf.edu.

\section{Recommended Citation}

Lepers, M.; Vexiau, R.; Bouloufa, N.; Dulieu, O.; and Kokoouline, Viatcheslav, "Photoassociation of a coldatom-molecule pair. II. Second-order perturbation approach" (2011). Faculty Bibliography 2010s. 1533. https://stars.library.ucf.edu/facultybib2010/1533

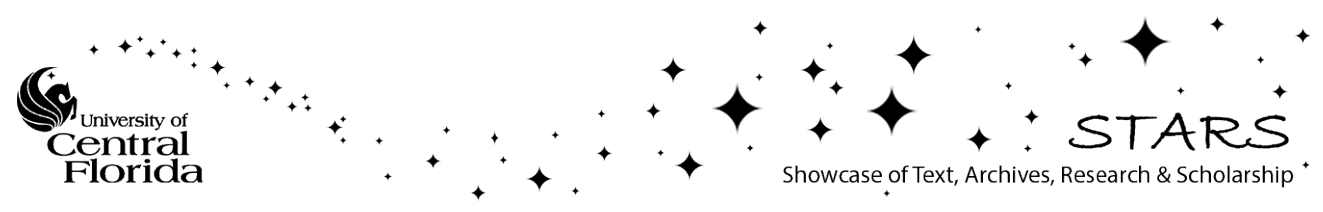




\title{
Photoassociation of a cold-atom-molecule pair. II. Second-order perturbation approach
}

\author{
M. Lepers,,${ }^{1,}$ R. Vexiau, ${ }^{1}$ N. Bouloufa, ${ }^{1}$ O. Dulieu, ${ }^{1}$ and V. Kokoouline ${ }^{1,2}$ \\ ${ }^{1}$ Laboratoire Aimé Cotton, CNRS, Bât. 505, Université Paris-Sud, F-91405 Orsay Cedex, France \\ ${ }^{2}$ Department of Physics, University of Central Florida, Orlando, Florida 32816, USA
}

(Received 3 January 2011; published 12 April 2011)

\begin{abstract}
The electrostatic interaction between an excited atom and a diatomic ground-state molecule in an arbitrary rovibrational level at large mutual separations is investigated with a general second-order perturbation theory, in the perspective of modeling the photoassociation between cold atoms and molecules. We find that the combination of quadrupole-quadrupole and van der Waals interactions competes with the rotational energy of the dimer, limiting the range of validity of the perturbative approach to distances larger than $100 \mathrm{Bohr}$ radii. Numerical results are given for the long-range interaction between $\mathrm{Cs}_{\text {and }} \mathrm{Cs}_{2}$, showing that the photoassociation is probably efficient for any $\mathrm{Cs}_{2}$ rotational energy.
\end{abstract}

DOI: 10.1103/PhysRevA.83.042707

PACS number(s): 34.50.Cx, 31.30.jh, 67.85.-d

\section{INTRODUCTION}

In a recent paper (hereafter referred to as paper I) [1] we investigated the electrostatic interaction between an atom and a diatomic molecule at large mutual separations. This kind of study is particularly relevant in the context of the amazing development of research on ultracold quantum gases, i.e., when the kinetic energy of the relative motion of the particles inside the gas is equivalent to a temperature far below $1 \mathrm{~K}$. Many enlightening review papers are available in the scientific literature, such as the most recent ones [2-4] devoted to ultracold molecular gases, which is our main purpose. Motivations and applications of such researches are thoroughly discussed in these reviews, and extensive bibliography can be found there, that we will not repeat in the present paper. Briefly, dilute atomic and molecular gases at ultracold temperatures exhibit pure quantum effects, as their dynamics is sensitive to quantum resonances and quantum statistics leading to Bose or Fermi degeneracy. Ultracold gases exhibit unique physical conditions for high-precision measurements related to the limit of validity of fundamental theories like the Standard Model or for quantum simulation of condensed matter phases such as superfluidity or superconductivity.

At such a low-energy regime, the relative motion of the particles inside the gas is controlled by their weak mutual interactions at large distance $R$, primarily induced by electrostatic forces described by a multipolar expansion in $R^{-n}$ terms. For instance, it is well known that spin-free or rotationless particles in their ground state interact mainly through van der Waals potentials varying as $C_{6} / R^{6}$ resulting from a secondorder perturbation treatment of the dipole-dipole interaction. Calculations of the long-range dispersion coefficients $C_{6}$ for alkali-metal or alkaline-earth-metal atoms-which represent the species of choice for ultracold gases-have nowadays reached an unprecedented accuracy [5,6] and represent an invaluable input for interpreting experiments. First-order terms may become dominant when one atom or both atoms are excited, resulting into interactions varying as $R^{-3}$ [7] or $R^{-5}$ [8] for dipole-dipole and quadrupole-quadrupole interactions, respectively.

*maxence.lepers@u-psud.fr
In principle, such calculations can be easily extended to the interaction between atoms and molecules with internal rotation. However, most previously published studies have been restricted to the situation where molecules are fixed in space $[9,10]$. A recent study actually treated the van der Waals interaction between two identical ground-state molecules in a given rotational level [11] using the second-order perturbation theory. In paper I, we were interested in the interaction between an excited atom and a ground-state molecule in a rotational level, governed by a first-order quadrupolequadrupole term varying as $R^{-5}$, that we evaluated through first-order degenerate perturbation theory. We demonstrated that this term competes with the rotational energy of the dimer, so that avoided crossings, and possibly long-range wells, could be expected in the long-range potential curves of the atom-dimer complex. Such patterns are relevant in the perspective of future studies aiming at associating an ultracold atom-molecule pair using laser light (photoassociation [PA]) to create stable ultracold triatomic molecules, according, for instance, to the reaction

$$
\mathrm{Cs}\left(6^{2} P\right)+\mathrm{Cs}_{2}\left(X^{1} \Sigma_{g}^{+}\right) \rightarrow \mathrm{Cs}_{3}^{*} \rightarrow \mathrm{Cs}_{3}+\text { photon. }
$$

In the present work, we extend our treatment to the second order of the perturbation expansion, to determine the next term varying as $R^{-6}$, which is expected to compete with the $R^{-5}$ term when $R$ decreases. As in paper I, we illustrate our development for the case of an alkali-metal ground-state $X^{1} \Sigma_{g}^{+}$molecule in an arbitrary rotational level $j$ with an alkali-metal atom excited to the $n^{2} P$ electronic level, but it can be easily generalized to arbitrary species. In Sec. II we first recall the general expression for the long-range multipolar expansion and the expression of the $C_{6}$ coefficients in the case of the van der Waals interaction between an atom and a molecule, and we relate these quantities to the dipole polarizabilities in imaginary frequencies, which are evaluated in Sec. III. Potential curves for the long-range interaction between an excited cesium atom and a ground-state $\mathrm{Cs}_{2}$ molecule are displayed in Sec. IV before concluding remarks and perspectives (Sec. V). Atomic units (a.u.) for distances $(1$ a.u. $=0.0529177 \mathrm{~nm})$ and for energies $(1$ a.u. $=$ $219474.63137 \mathrm{~cm}^{-1}$ ) will be used throughout the paper, except otherwise stated. 


\section{EXPRESSION OF THE VAN DER WAALS INTERACTION}

We first recall briefly the notation used in paper I. The electrostatic potential energy between two charge distributions $A$ (the dimer) and $B$ (the atom) at large distance $R$ (i.e., beyond the LeRoy radius [12]) is expressed as the usual multipolar expansion

$$
\begin{aligned}
\hat{V}_{A B}(R)= & \sum_{L_{A}, L_{B}=0}^{+\infty} \sum_{M=-L_{<}}^{L_{<}} \frac{1}{R^{1+L_{A}+L_{B}}} \\
& \times f_{L_{A} L_{B} M} \hat{Q}_{L_{A}}^{M}\left(\hat{r}_{A}\right) \hat{Q}_{L_{B}}^{-M}\left(\hat{r}_{B}\right),
\end{aligned}
$$

where $\hat{Q}_{L_{X}}^{M}\left(\hat{r}_{X}\right)$ is the operator associated with the $2^{L_{X}}$ pole of charge distribution $X(X=A$ or $B)$, expressed in the coordinate system with the origin at the center of mass of $X$

$$
\hat{Q}_{L_{X}}^{M}\left(\hat{r}_{X}\right)=\sqrt{\frac{4 \pi}{2 L_{X}+1}} \sum_{i \in X} q_{i} \hat{r}_{i}^{L_{X}} Y_{L_{X}}^{M}\left(\hat{\theta}_{i}, \hat{\phi}_{i}\right),
$$

where

$$
\begin{aligned}
f_{L_{A} L_{B} M}= & \frac{(-1)^{L_{B}}\left(L_{A}+L_{B}\right) !}{\sqrt{\left(L_{A}+M\right) !\left(L_{A}-M\right) !}} \\
& \times \frac{1}{\sqrt{\left(L_{B}+M\right) !\left(L_{B}-M\right) !}}
\end{aligned}
$$

and $L_{<}$is the minimum of $L_{A}$ and $L_{B}$. The $Z$ quantization axis for the projections $\pm M$ in the above equations is oriented from $A$ to $B$, yielding the $(-1)^{L_{B}}$ factor in Eq. (4). We define two body-fixed coordinate systems (CS) displayed in Fig. 1: the dimer CS (or D-CS) with axes $X_{A}, Y_{A}, Z_{A}$, and the trimer CS (or T-CS) with axes $X, Y, Z$. The T-CS is deduced from the D-CS by a rotation with an angle $\delta$ around the $Y$ axis. The T-CS is related to the space-fixed coordinate system $(\tilde{x} \tilde{y} \tilde{z})$ by the usual Euler angles $(\alpha, \beta, \gamma)$.

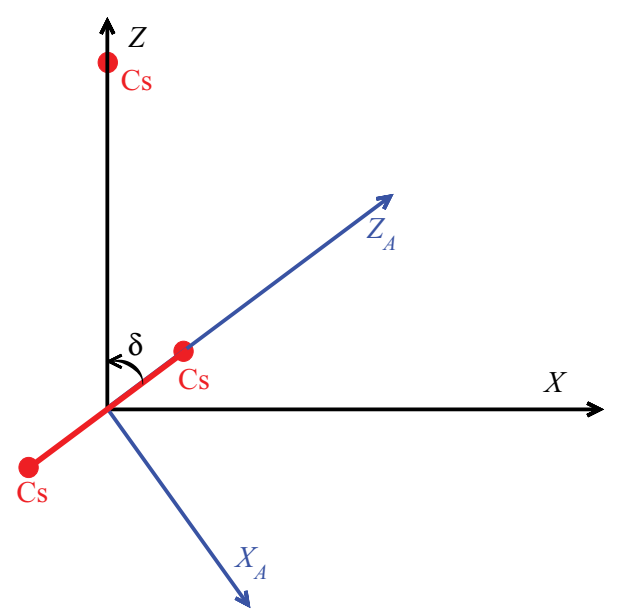

FIG. 1. (Color online) The dimer $\left[X_{A} Y_{A} Z_{A}\right]$ (D-CS), and trimer [XYZ] (T-CS) coordinate systems. The $Z_{A}$ axis is along the dimer axis, while $Z$ is oriented from the center of mass of the dimer toward the atom $B$. The $Y$ and $Y_{A}$ axes coincide and point into the plane of the figure. The T-CS is deduced from the D-CS by a rotation with an angle $\delta$ about the $Y$ axis. The Euler angles $(\alpha, \beta, \gamma)$ are not represented here.
The dimer is assumed in an arbitrary vibrational $\left|v_{d}\right\rangle$ and rotational state $|j\rangle$ of its electronic ground state $\left|X^{1} \Sigma_{g}^{+}\right\rangle$. The atom $B$ is chosen with a single outer electron being excited to the $p$ state $|n, \ell=1\rangle$. The projections $m_{j}$ and $\lambda$ of $j$ and $\ell$ are defined with respect to the $Z$ axis. The energy origin corresponds to an infinite separation between the atom and the dimer. The first-order perturbation theory yields the quadrupole-quadrupole energies with zeroth-order wave functions that can be written as a linear combination of the various $\left|m_{j}, \lambda\right\rangle$ substates of the complex

$$
\left|\Phi_{0}^{0}\right\rangle=\sum_{m_{j} \lambda} c_{m_{j} \lambda}\left|m_{j}, \lambda\right\rangle
$$

where $c_{m_{j} \lambda}$ are real. The quantity $m_{J}=m_{j}+\lambda$ is the projection of the total angular momentum $J$ on the trimer axis, which is the only strictly good quantum number of the problem. In the following, we will not refer to the values of $J$, as the rotation of the trimer is not introduced. Therefore $m_{J}$ is also conserved.

The $C_{6}$ or van der Waals coefficient comes from dipoledipole interaction whose expression $\hat{V}_{A B}^{d d}(R)$ is obtained by setting $L_{A}=L_{B}=1$ in Eq. (2):

$$
\hat{V}_{A B}^{d d}(R)=-\frac{2}{R^{3}} \sum_{M=-1}^{1} \frac{\hat{Q}_{1}^{M}\left(\hat{r}_{A}\right) \hat{Q}_{1}^{-M}\left(\hat{r}_{B}\right)}{(1+M) !(1-M) !} .
$$

As the permanent dipole of both the atom and the dimer are zero for any states of the atom and the molecule, the (nondegenerate) second-order perturbation theory is used to obtain the related correction written as $C_{6} / R^{6}$ with

$$
\begin{aligned}
C_{6}= & -4 \sum_{a, b \neq 0} \frac{1}{\left(E_{a}^{0}-E_{A 0}^{0}\right)+\left(E_{b}^{0}-E_{B 0}^{0}\right)} \\
& \times \sum_{M=-1}^{1} \frac{\left\langle\Phi_{A 0}^{0}\left|\hat{Q}_{1}^{M}\right| \Phi_{a}^{0}\right\rangle\left\langle\Phi_{B 0}^{0}\left|\hat{Q}_{1}^{-M}\right| \Phi_{b}^{0}\right\rangle}{(1+M) !(1-M) !} \\
& \times \sum_{M^{\prime}=-1}^{1} \frac{\left\langle\Phi_{a}^{0}\left|\hat{Q}_{1}^{-M^{\prime}}\right| \Phi_{A 0}^{0}\right\rangle\left\langle\Phi_{b}^{0}\left|\hat{Q}_{1}^{M^{\prime}}\right| \Phi_{B 0}^{0}\right\rangle}{\left(1+M^{\prime}\right) !\left(1-M^{\prime}\right) !} .
\end{aligned}
$$

For a given $\left|\Phi_{0}^{0}\right\rangle$, the corresponding $C_{6}$ coefficient can be expanded as a linear combination of crossed terms

$$
C_{6}=\sum_{m_{j_{1}} \lambda_{1}} \sum_{m_{j_{2}} \lambda_{2}} c_{m_{j_{1}} \lambda_{1}} c_{m_{j_{2}} \lambda_{2}} C_{6}^{\mathrm{cr}},
$$

where the crossed coefficients $C_{6}^{\mathrm{cr}}$ are

$$
\begin{aligned}
C_{6}^{\mathrm{cr}}= & -4 \sum_{a, b} \sum_{M, M^{\prime}} \frac{1}{\left(E_{a}^{0}-E_{X v_{d} j}^{0}\right)+\left(E_{b}^{0}-E_{n \ell}^{0}\right)} \\
& \times \frac{\left\langle X v_{d} j m_{j_{1}}\left|\hat{Q}_{1}^{M}\right| \Phi_{a}^{0}\right\rangle\left\langle n \ell \lambda_{1}\left|\hat{Q}_{1}^{-M}\right| \Phi_{b}^{0}\right\rangle}{(1+M) !(1-M) !} \\
& \times \frac{\left\langle\Phi_{a}^{0}\left|\hat{Q}_{1}^{-M^{\prime}}\right| X v_{d} j m_{j_{2}}\right\rangle\left\langle\Phi_{b}^{0}\left|\hat{Q}_{1}^{M^{\prime}}\right| n \ell \lambda_{2}\right\rangle}{\left(1+M^{\prime}\right) !\left(1-M^{\prime}\right) !}
\end{aligned}
$$

The summation is performed over all possible states $\Phi_{a}^{0}$ and $\Phi_{b}^{0}$ of the $A$ and $B$ systems.

With the approach of imaginary frequencies dipole polarizabilities (see, e.g., Ref. [13]), the sum in Eq. (9) can be 
factorized into separated contributions from $A$ and $B$. For this purpose, we use the identity

$$
\frac{1}{|x|+|y|}=\frac{2}{\pi} \int_{0}^{+\infty} d \omega \frac{|x||y|}{\left(x^{2}+\omega^{2}\right)\left(y^{2}+\omega^{2}\right)}
$$

to transform the first term of Eq. (9), with $|x|=E_{a}^{0}-$ $E_{X v_{d} j}^{0}$ and $|y|=E_{b}^{0}-E_{n \ell}^{0}$. This approach is applicable if $E_{a}^{0}-E_{X v_{d} j}^{0}>0$ and $E_{b}^{0}-E_{n \ell}^{0}>0$. This is always the case for the dimer if it is in its ground rovibronic state. Moreover, if the dimer is homonuclear, radiative transitions between rovibrational levels belonging to the same electronic state are forbidden, so that the identity still holds for any rovibrational level of the $X^{1} \Sigma_{g}^{+}$state. In contrast, atom $B$ is in its first electronically excited state. Therefore, taking cesium as an example, Eq. (10) is correct for all transitions, except $6^{2} P \rightarrow 6^{2} S$, for which $E_{b}^{0}-E_{n \ell}^{0}<0$. In the latter case, a similar factorization can be performed, by setting $|y|=-E_{b}^{0}+E_{n \ell}^{0}$, and by using the following identity:

$$
\begin{aligned}
\frac{1}{|x|-|y|} & =-\frac{1}{|x|+|y|}+\frac{2|x|}{x^{2}-y^{2}} \\
& =\frac{2}{\pi} \int_{0}^{+\infty} d \omega \frac{x y}{\left(x^{2}+\omega^{2}\right)\left(y^{2}+\omega^{2}\right)}+\frac{2 x}{x^{2}-y^{2}} .
\end{aligned}
$$

Inserting Eqs. (10) and (11) into Eq. (9), and following Ref. [14], we obtain

$$
\begin{aligned}
C_{6}^{\mathrm{cr}}= & -\sum_{M=-1}^{1} \sum_{M^{\prime}=-1}^{1} \frac{4}{(1+M) !(1-M) !\left(1+M^{\prime}\right) !\left(1-M^{\prime}\right) !} \\
& \times\left[\frac{1}{2 \pi} \int_{0}^{+\infty} d \omega \alpha_{M M^{\prime}}^{m_{j_{1}} m_{j_{2}}}(i \omega) \alpha_{-M-M^{\prime}}^{\lambda_{1} \lambda_{2}}(i \omega)\right. \\
& +\sum_{b} \Theta\left(-\Delta E_{b}^{0}\right) \alpha_{M M^{\prime}}^{m_{j_{1}} m_{j_{2}}}\left(\omega=\Delta E_{b}^{0}\right) \\
& \left.\times\left\langle n \ell \lambda_{1}\left|\hat{Q}_{1}^{-M}\right| \Phi_{b}^{0}\right\rangle\left\langle\Phi_{b}^{0}\left|\hat{Q}_{1}^{M^{\prime}}\right| n \ell \lambda_{2}\right\rangle\right],
\end{aligned}
$$

where $\Delta E_{b}^{0}=E_{b}^{0}-E_{n \ell}^{0}$, and $\Theta(x)$ is the Heaviside function, equal to 1 for each downward transition and 0 otherwise. The expression of dipole polarizabilities is generalized to arbitrary frequencies (real or imaginary) according to

$$
\begin{aligned}
\alpha_{M M^{\prime}}^{m_{j_{1}} m_{j_{2}}}(z)= & 2(-1)^{M} \sum_{a} \frac{\left(E_{a}^{0}-E_{X v_{d} j}^{0}\right)}{\left(E_{a}^{0}-E_{X v_{d} j}^{0}\right)^{2}-z^{2}} \\
& \times\left\langle X v_{d} j m_{j_{1}}\left|\hat{Q}_{1}^{M}\right| \Phi_{a}^{0}\right\rangle,\left\langle\Phi_{a}^{0}\left|\hat{Q}_{1}^{-M^{\prime}}\right| X v_{d} j m_{j_{2}}\right\rangle
\end{aligned}
$$

$$
\begin{aligned}
\alpha_{-M-M^{\prime}}^{\lambda_{1} \lambda_{2}}(z)= & 2(-1)^{M} \sum_{b} \frac{\left(E_{b}^{0}-E_{n \ell}^{0}\right)}{\left(E_{b}^{0}-E_{n \ell}^{0}\right)^{2}-z^{2}} \\
& \times\left\langle n \ell \lambda_{1}\left|\hat{Q}_{1}^{-M}\right| \Phi_{b}^{0}\right\rangle\left\langle\Phi_{b}^{0}\left|\hat{Q}_{1}^{M^{\prime}}\right| n \ell \lambda_{2}\right\rangle,
\end{aligned}
$$

where $z$ can be real or imaginary. In Eq. (12) the first term inside the brackets is the well-known product of the polarizabilities at imaginary frequencies of the molecular $\left|X v_{d} j\right\rangle$ and atomic $|n \ell\rangle$ states (the corresponding labels are omitted for clarity sake). In the second term appears the polarizability of the dimer at the real frequency of each downward transition of the atom. We note that if the dimer polarizability is negative and significantly large, the second term in the square brackets can become larger in magnitude than the first term and, therefore, can turn the $C_{6}$ sign to positive.

\section{CALCULATION OF POLARIZABILITIES}

\section{A. The polarizability of the dimer}

Molecular polarizabilities are most often calculated ignoring rotation, so that it is wise to separate it in the equations of Sec. II. We start by expressing the dipole matrix element $\left\langle X \Lambda v_{d} j m_{j}\left|\hat{Q}_{1}^{M}\right| \Phi_{a}^{0}\right\rangle$ of Eq. (13) between the states defined with respect to the T-CS, in terms of matrix elements between states defined in the D-CS. For clarity, we explicitly write the dimer quantum number $\Lambda$, which is the projection of the electronic angular momentum on the $Z_{A}$ axis. Specifying as well all the relevant quantum numbers according to $\left|\Phi_{a}^{0}\right\rangle=\left|X^{\prime} \Lambda^{\prime} v_{d}^{\prime} j^{\prime} m_{j}^{\prime}\right\rangle$, we have

$$
\begin{aligned}
\langle X & \left.\Lambda v_{d} j m_{j}\left|\hat{Q}_{1}^{M}\right| \Phi_{a}^{0}\right\rangle \\
& =\sum_{\mu}\left\langle X \Lambda v_{d} j m_{j}\left|d_{M \mu}^{1}(\delta) \hat{q}_{1}^{\mu}\right| X^{\prime} \Lambda^{\prime} v_{d}^{\prime} j^{\prime} m_{j}^{\prime}\right\rangle \\
& =\sum_{\mu}\left\langle j m_{j}\left|d_{M \mu}^{1}\right| j^{\prime} m_{j}^{\prime}\right\rangle\left\langle X \Lambda v_{d}\left|\hat{q}_{1}^{\mu}\right| X^{\prime} \Lambda^{\prime} v_{d}^{\prime}\right\rangle,
\end{aligned}
$$

where the quantities $\hat{q}_{1}^{\mu}$ are the components of the electric dipole moment defined with respect to the D-CS. The index $\mu$ is either 0 for $\Sigma \rightarrow \Sigma$ transitions $\left(\Lambda=\Lambda^{\prime}=0\right)$, or \pm 1 for $\Sigma \rightarrow \Pi$ transitions. In the T-CS the rotational wave function of the dimer is proportional to $d_{m_{j} 0}^{j}$ and to $d_{m_{j} \pm 1}^{j}$, for $\Sigma$ and $\Pi$ states, respectively. Using the properties of the $d_{\mu \nu}^{j}$ rotation matrices [15], we find

$$
\left\langle j m_{j}\left|d_{M \mu}^{1}\right| j^{\prime} m_{j}^{\prime}\right\rangle=\sqrt{\frac{2 j^{\prime}+1}{2 j+1}} C_{1 M j^{\prime} m_{j}^{\prime}}^{j m_{j}} C_{1 \mu j^{\prime}-\mu}^{j 0},
$$

where the $C_{1 M j^{\prime} m_{j}^{\prime}}^{j m_{j}}$ and $C_{1 \mu j^{\prime}-\mu}^{j 0}$ are Clebsch-Gordan coefficients. If in Eq. (13) we neglect the rotational part of the $0^{\text {th }}$ order energy (only low rotational levels are relevant for the cold temperature domain), i.e.,

$$
E_{X v_{d} j}^{0}=E_{X v_{d}}^{0}+B_{v_{d}} j(j+1) \approx E_{X v_{d}}^{0},
$$

we identify the exact expressions of the parallel polarizability $\alpha_{\|}$and of the perpendicular polarizability $\alpha_{\perp}$ with respect to the $Z_{A}$ axis, for $\mu=0$ and \pm 1 , respectively. Therefore, Eq. (13) becomes

$$
\begin{aligned}
\alpha_{M M^{\prime}}^{m_{j_{1}} m_{j_{2}}}(z) \approx & \sum_{j^{\prime}, m_{j}^{\prime}} \frac{2 j+1}{2 j^{\prime}+1}\left[\left(C_{10 j 0}^{j^{\prime} 0}\right)^{2} \alpha_{\|}(z)\right. \\
& \left.+2\left(C_{11 j 0}^{j^{\prime} 1}\right)^{2} \alpha_{\perp}(z)\right] C_{1-M j m_{j_{1}}}^{j^{\prime} m_{j}^{\prime}} C_{1-M^{\prime} j m_{j_{2}}}^{j^{\prime} m_{j}^{\prime}}
\end{aligned}
$$

where we used the identity [15]

$$
C_{a \alpha b \beta}^{c \gamma}=(-1)^{a-\alpha} \sqrt{\frac{2 c+1}{2 b+1}} C_{c \gamma a-\alpha}^{b \beta}
$$


in order to put all the primes in the upper indices. The dependence on the rotational state of the dimer is restricted to the coefficients of the vibronic polarizabilities of the dimer. We note that Eq. (18) is valid for real, imaginary, and zero frequencies (i.e., for static polarizabilities).

\section{B. The valence contribution to the atomic polarizability}

We perform a similar development for the atom, in order to separate the radial and angular parts of the matrix elements of the dipole moment. By writing explicitly $\left|\Phi_{b}^{0}\right\rangle=\left|n^{\prime} \ell^{\prime} \lambda^{\prime}\right\rangle$ and by using Eq. (19), we rewrite the dipole matrix elements as

$$
\left\langle n \ell \lambda\left|\hat{Q}_{1}^{-M}\right| n^{\prime} \ell^{\prime} \lambda^{\prime}\right\rangle=(-1)^{M} \sqrt{\frac{2 \ell+1}{2 \ell^{\prime}+1}} C_{10 \ell 0}^{\ell^{\prime} 0} C_{1 M \ell \lambda}^{\ell^{\prime} \lambda^{\prime}} r_{n \ell n^{\prime} \ell^{\prime}},
$$

where $r_{n \ell n^{\prime} \ell^{\prime}}=\left\langle n \ell|r| n^{\prime} \ell^{\prime}\right\rangle$ is the matrix element of the valence electron radial coordinate. Equation (14) becomes

$$
\begin{aligned}
\alpha_{-M-M^{\prime}}^{\lambda_{1} \lambda_{2}}(z)= & 2 \sum_{n^{\prime}, \ell^{\prime}} \frac{\left(E_{n^{\prime} \ell^{\prime}}^{0}-E_{n \ell}^{0}\right)}{\left(E_{n^{\prime} \ell^{\prime}}^{0}-E_{n \ell}^{0}\right)^{2}-z^{2}} \\
& \times r_{n \ell n^{\prime} \ell^{\prime}}^{2} \frac{2 \ell+1}{2 \ell^{\prime}+1}\left(C_{10 \ell 0}^{\ell^{\prime} 0}\right)^{2} \\
& \times \sum_{\lambda^{\prime}} C_{1 M \ell \lambda_{1}}^{\ell^{\prime} \lambda^{\prime}} C_{1 M^{\prime} \ell \lambda_{2}}^{\ell^{\prime} \lambda^{\prime}} .
\end{aligned}
$$

The index $\ell^{\prime}$ above takes values corresponding to dipoleallowed transitions, i.e., $\ell^{\prime}=0,2$ in the present case of a $\operatorname{Cs}\left(6^{2} P\right)$ atom, and $n^{\prime}$ for all the relevant atomic levels.

The similarity between Eqs. (18) and (21) confirms the equivalence in the formalism between the rotational angular momentum of the dimer and the electronic orbital momentum of the atom, which makes the generalization to more complicated cases such as molecule-molecule long-range interactions quite straightforward. However, it is not possible to express Eq. (21) as a function of the sole polarizability of the atomic $n \ell$ level. For instance, if $\ell=1, M^{\prime}=M$, and $\lambda_{2}=\lambda_{1}$, the dipole polarizability $\alpha_{M M}^{\lambda_{1} \lambda_{1}}(z)$ of the sublevel $n P \lambda_{1}$ contains angular factors that are different for $P \rightarrow S$ and $P \rightarrow D$ transitions [14]. In the usual definition of the polarizability, an average is made over all the sublevels $\lambda_{1}$ leading to the disappearance of the angular factors, which is not the case here. Therefore, we introduce state-to-state polarizabilities $\alpha_{n \ell n^{\prime} \ell^{\prime}}$ associated to the different $n \ell \rightarrow n^{\prime} \ell^{\prime}$ transitions

$$
\alpha_{n \ell n^{\prime} \ell^{\prime}}(z)=\frac{2}{3} \frac{\left(E_{n^{\prime} \ell^{\prime}}^{0}-E_{n \ell}^{0}\right)}{\left(E_{n^{\prime} \ell^{\prime}}^{0}-E_{n \ell}^{0}\right)^{2}-z^{2}} r_{n \ell n^{\prime} \ell^{\prime}}^{2}\left(C_{10 \ell 0}^{\ell^{\prime} 0}\right)^{2},
$$

so that Eq. (21) becomes

$$
\begin{aligned}
\alpha_{-M-M^{\prime}}^{\lambda_{1} \lambda_{2}}(z)= & 3 \sum_{\ell^{\prime}=\{\ell-1, \ell+1\}} \frac{2 \ell+1}{2 \ell^{\prime}+1} \sum_{n^{\prime}} \alpha_{n \ell n^{\prime} \ell^{\prime}}(z) \\
& \times \sum_{\lambda^{\prime}} C_{1 M \ell \lambda_{1}}^{\ell^{\prime} \lambda^{\prime}} C_{1 M^{\prime} \ell \lambda_{2}}^{\ell^{\prime} \lambda^{\prime}} .
\end{aligned}
$$

The state-to-state polarizabilities obey the property $\sum_{n^{\prime} \ell^{\prime}} \alpha_{n \ell n^{\prime} \ell^{\prime}} \approx \alpha_{n \ell}$, with $\alpha_{n \ell}$ being the actual (isotropic) atomic polarizability of the level $n \ell$. This identity is only approximate as the effect of core electrons have been neglected so far.

\section{The core contribution to the atomic polarizability}

Following Ref. [6] we assume that the contribution of the core electrons can be treated as an additional correction $\alpha_{c}$ to the total polarizability $\bar{\alpha}_{n \ell}$, independent of the atomic state $|n \ell\rangle$ :

$$
\bar{\alpha}_{n \ell}=\sum_{n^{\prime} \ell^{\prime}} \alpha_{n \ell n^{\prime} \ell^{\prime}}+\alpha_{c} .
$$

In our numerical calculations, $\alpha_{c}$ is obtained in the following way. First, the contribution of the valence electron is evaluated using tabulated values of dipole moments for transitions from the $6^{2} S$ state of cesium. The result is then compared to the polarizability of Ref. [6], which accounts for the ionic core contribution, and the difference between our result and that of Ref. [6] is then attributed to $\alpha_{c}$.

It is not straightforward to see that the core polarizability brings a simple additive contribution to the $C_{6}$ coefficients. As inner shells have different angular quantum numbers, we first consider only the electrons of a given closed inner shell, e.g., $4 d$ for cesium. A sum over all the closed shells will be taken at the end. We note $n_{c}, \ell_{c}, k_{i}, \sigma_{i}$ the principal, orbital, azimuthal, and spin quantum numbers of the $i$ th electron of the shell. The indexes $n_{c}$ and $\ell_{c}$ are identical for the $2\left(2 \ell_{c}+1\right)$ electrons, whereas $k_{i}$ varies from $-\ell_{c}$ to $+\ell_{c}$, and $\sigma_{i}= \pm \frac{1}{2}$. Since the dipole operator is monoelectronic, the ionic core brings to the $C_{6}$ coefficient an additional term $C_{6}^{c}$ which is the sum of all contributions $C_{6}^{n_{c} l_{c}}$ of every shell $n_{c}, l_{c}$

$$
C_{6}^{n_{c} l_{c}}=\sum_{i \in \text { shell }} C_{6}^{n_{c} l_{c}}(i)
$$

where the general expression for $C_{6}^{n_{c} l_{c}}(i)$ is

$$
\begin{aligned}
C_{6}^{n_{c} l_{c}}(i)= & -4 \sum_{a, b \neq 0} \sum_{M, M^{\prime}} \frac{1}{\left(E_{a}^{0}-E_{A 0}^{0}\right)+\left(E_{b}^{0}-E_{B 0}^{0}\right)} \\
& \times \frac{\left\langle\Phi_{A 0}^{0}\left|\hat{Q}_{1}^{M}\right| \Phi_{a}^{0}\right\rangle\left\langle\Phi_{B 0}^{0}\left|\hat{Q}_{1}^{-M}(i)\right| \Phi_{b i}^{0}\right\rangle}{(1+M) !(1-M) !} \\
& \times \frac{\left\langle\Phi_{a}^{0}\left|\hat{Q}_{1}^{-M^{\prime}}\right| \Phi_{A 0}^{0}\right\rangle\left\langle\Phi_{b i}^{0}\left|\hat{Q}_{1}^{M^{\prime}}(i)\right| \Phi_{B 0}^{0}\right\rangle}{\left(1+M^{\prime}\right) !\left(1-M^{\prime}\right) !} .
\end{aligned}
$$

In Eq. (26), the atomic states are characterized by independent electrons

$$
\begin{aligned}
\left|\Phi_{B 0}^{0}\right\rangle= & \left|n_{c} \ell_{c},-\ell_{c},-\frac{1}{2}\right\rangle \times \cdots\left|n_{c} \ell_{c}, k_{i}, \sigma_{i}\right\rangle \\
& \times \cdots \times\left|n_{c} \ell_{c}, \ell_{c}, \frac{1}{2}\right\rangle|n \ell \lambda\rangle,
\end{aligned}
$$

and similarly $\left|\Phi_{b i}^{0}\right\rangle=\left|n_{c} \ell_{c},-\ell_{c},-\frac{1}{2}\right\rangle \ldots\left|n_{c}^{\prime} \ell_{c}^{\prime}, k_{i}^{\prime}, \sigma_{i}\right\rangle \ldots$ $\left|n_{c}^{\prime} \ell_{c}^{\prime}, \ell_{c}^{\prime}, \frac{1}{2}\right\rangle|n \ell \lambda\rangle$, and the molecular states, $\left|\Phi_{A 0}^{0}\right\rangle$ and $\left|\Phi_{a}^{0}\right\rangle$, have the same quantum numbers as previously. The states $\left|\Phi_{B 0}^{0}\right\rangle$ and $\left|\Phi_{b i}^{0}\right\rangle$ are antisymmetric with respect to the 
permutation of two electrons. However, for simplicity, they are written here as simple products of the individual electronic states, which does not modify the value of $C_{6}^{n_{c} l_{c}}$. In Eq. (26), the energy $E_{b}^{0}$ is independent on the index $i$, since $i$ makes a distinction only between different projection of orbital and spin angular momenta.

Unlike the valence electron, as the core is not affected by the $C_{5}$ coefficient, there is no crossed term in the calculation of $C_{6}^{n_{c} l_{c}}(i)$. As a consequence, the Wigner-Eckart theorem imposes $m_{j}^{\prime}+M=m_{j}$ and $m_{j}^{\prime}+M^{\prime}=m_{j}$, as well as $k_{i}^{\prime}-$ $M=k_{i}$ and $k_{i}^{\prime}+M^{\prime}=k_{i}$, hence the condition $M^{\prime}=M$. So the double summation of Eq. (26) reduces to a single one over M. Similarly to Eq. (12), we can factorize Eq. (26) with polarizability-like quantities. Because there is no allowed transitions from the last inner shell of the atom to lower-energy states, that factorization does not contain any additional term due to the excited state. It yields

$$
\begin{aligned}
C_{6}^{n_{c} l_{c}}= & -\frac{2}{\pi} \sum_{M=-1}^{1} \frac{1}{[(1+M) !(1-M) !]^{2}} \\
& \times \int_{0}^{+\infty} d \omega \alpha_{M M}^{m_{j} m_{j}}(i \omega) \alpha_{-M-M}^{n_{c} l_{c}}(i \omega),
\end{aligned}
$$

where $\alpha_{M M}^{m_{j} m_{j}}$ is given by Eq. (18) and $\alpha_{-M-M}^{n_{c} l_{c}}$ is similar to Eq. (21):

$$
\begin{aligned}
\alpha_{-M-M}^{n_{c} l_{c}}(i \omega)= & 2 \sum_{n_{c}^{\prime} \ell_{c}^{\prime}} \frac{E_{n_{c}^{\prime} \ell_{c}^{\prime}}^{0}-E_{n_{c} \ell_{c}}^{0}}{\left(E_{n_{c}^{\prime} \ell_{c}^{\prime}}^{0}-E_{n_{c} \ell_{c}}^{0}\right)^{2}+\omega^{2}} \\
& \times \frac{2 \ell_{c}+1}{2 \ell_{c}^{\prime}+1}\left(C_{10 \ell_{c} 0}^{\ell_{c}^{\prime} 0}\right)^{2}\left(r_{n_{c} \ell_{c} n_{c}^{\prime} \ell_{c}^{\prime}}\right)^{2} \\
& \times \sum_{k k^{\prime} \sigma \sigma^{\prime}}\left(C_{1 M \ell_{c} k}^{\ell_{c}^{\prime} k^{\prime}}\right)^{2} \delta_{\sigma \sigma^{\prime}} .
\end{aligned}
$$

In Eq. (29), the sum over $k$ and $\sigma$ runs over all the core electrons $i$. The sum over the two different spin projections for each orbital gives factor of 2 . The sum of the ClebschGordan coefficients yields $\frac{2 \ell_{c}^{\prime}+1}{3}$. Therefore, the angular part of the dipole moment disappears, and $\alpha_{-M-M}^{c}$ finally reads

$$
\begin{aligned}
\alpha_{-M-M}^{n_{c} l_{c}}(i \omega)= & 4 \sum_{n_{c}^{\prime} \ell_{c}^{\prime}} \frac{E_{n_{c}^{\prime} \ell_{c}^{\prime}}^{0}-E_{n_{c} \ell_{c}}^{0}}{\left(E_{n_{c}^{\prime} \ell_{c}^{\prime}}^{0}-E_{n_{c} \ell_{c}}^{0}\right)^{2}+\omega^{2}} \\
& \times \frac{2 \ell_{c}+1}{3}\left(C_{10 \ell_{c} 0}^{\ell_{c}^{\prime} 0}\right)^{2}\left(r_{n_{c} \ell_{c} n_{c}^{\prime} \ell_{c}^{\prime}}\right)^{2} .
\end{aligned}
$$

As one can see from the above expression, it is not necessary to separate different series of transitions $\ell_{c} \rightarrow \ell_{c}^{\prime}$, and we obtain a meaningful polarizability. Finally, we can extend this results to all inner shells that gives the core polarizability:

$$
\begin{aligned}
\alpha_{c}(i \omega)= & 4 \sum_{n_{c} \ell_{c} n_{c}^{\prime} \ell_{c}^{\prime}} \frac{E_{n_{c}^{\prime} \ell_{c}^{\prime}}^{0}-E_{n_{c} \ell_{c}}^{0}}{\left(E_{n_{c}^{\prime} \ell_{c}^{\prime}}^{0}-E_{n_{c} \ell_{c}}^{0}\right)^{2}+\omega^{2}} \\
& \times \frac{2 \ell_{c}+1}{3}\left(C_{10 \ell_{c} 0}^{\ell_{c}^{\prime} 0}\right)^{2}\left(r_{n_{c} \ell_{c} n_{c}^{\prime} \ell_{c}^{\prime}}\right)^{2},
\end{aligned}
$$

and the core contribution $C_{6}^{c}$ to the $C_{6}$ coefficient:

$$
\begin{aligned}
C_{6}^{c}= & -\frac{2}{\pi} \sum_{M} \frac{1}{[(1+M) !(1-M) !]^{2}} \\
& \times \sum_{j^{\prime} m_{j^{\prime}}} \frac{2 j+1}{2 j^{\prime}+1}\left(C_{1 M j m_{j}}^{j^{\prime} m_{j}^{\prime}}\right)^{2} \int d \omega \alpha_{c}(i \omega) \\
& \times\left[\left(C_{10 j 0}^{j^{\prime} 0}\right)^{2} \alpha_{\|}(i \omega)+2\left(C_{11 j 0}^{j^{\prime} 1}\right)^{2} \alpha_{\perp}(i \omega)\right] .
\end{aligned}
$$

With the approximations above, we obtain an additional term that depends on the physical polarizabilities of the atomic core and the molecule, and in which the rotation of the dimer appears explicitly. As all the factors in Eq. (32) are positive, $C_{6}^{c}$ is negative, and, thus, it makes the interaction between the atom and the dimer more attractive (or less repulsive).

\section{General expression for $\boldsymbol{C}_{6}$}

We summarize all the results of the previous paragraphs. We recall that the $C_{6}$ coefficients are calculated for each eigenvector of the quadrupole-quadrupole operator. Each eigenvector $\left|\Phi_{0}^{0}\right\rangle$ is a linear combination of states $\left|m_{j}, \lambda\right\rangle$ [see Eq. (5)] with $m_{j}+\lambda$ being constant. For a given $\left|\Phi_{0}^{0}\right\rangle$, the general expression for $C_{6}$ is

$$
\begin{aligned}
C_{6}=- & 3 \sum_{m_{j_{1}} \lambda_{1}} \sum_{m_{j_{2}} \lambda_{2}} \sum_{M M^{\prime}} \sum_{j^{\prime} m_{j}^{\prime}} \sum_{\ell^{\prime} \lambda^{\prime}} \frac{c_{m_{j_{1}} \lambda_{1}} c_{m_{j_{2}} \lambda_{2}}}{(1+M) !(1-M) !\left(1+M^{\prime}\right) !\left(1-M^{\prime}\right) !} \frac{2 j+1}{2 j^{\prime}+1} C_{1-M j m_{j_{1}}}^{j^{\prime} m_{j}^{\prime}} C_{1-M^{\prime} j m_{j_{2}}}^{j^{\prime} m_{j}^{\prime}} \frac{2 \ell+1}{2 \ell^{\prime}+1} C_{1 M \ell \lambda_{1}}^{\ell^{\prime} \lambda^{\prime}} C_{1 M^{\prime} \ell \lambda_{2}}^{\ell^{\prime} \lambda^{\prime}} \\
& \times\left\{\frac{2}{\pi} \int_{0}^{+\infty} d \omega\left[\left(C_{10 j 0}^{j^{\prime} 0}\right)^{2} \alpha_{\|}(i \omega)+2\left(C_{11 j 0}^{j^{\prime} 1}\right)^{2} \alpha_{\perp}(i \omega)\right] \alpha_{\ell \ell^{\prime}}(i \omega)+4 \sum_{n^{\prime}} \Theta\left(-\Delta E_{n^{\prime} \ell^{\prime}, n \ell}^{0}\right)\left[\left(C_{10 j 0}^{j^{\prime} 0}\right)^{2} \alpha_{\|}\left(\Delta E_{n^{\prime} \ell^{\prime}, n \ell}^{0}\right)\right.\right. \\
& \left.\left.+2\left(C_{11 j 0}^{j^{\prime} 1}\right)^{2} \alpha_{\perp}\left(\Delta E_{n^{\prime} \ell^{\prime}, n \ell}^{0}\right)\right]\left(\mu_{n^{\prime} \ell^{\prime}, n \ell}\right)^{2}\right\}-\frac{2}{\pi} \sum_{m_{j_{1}} \lambda_{1}} \sum_{M} \sum_{j^{\prime} m_{j}^{\prime}} \frac{c_{m_{j_{1}} \lambda_{1}}^{2}}{[(1+M) !(1-M) !]^{2}} \frac{2 j+1}{2 j^{\prime}+1}\left(C_{1 M j m_{j 1} 1}^{j^{\prime} m_{j}^{\prime}}\right)^{2} \\
& \times \int_{0}^{+\infty} d \omega\left[\left(C_{10 j 0}^{j^{\prime} 0}\right)^{2} \alpha_{\|}(i \omega)+2\left(C_{11 j 0}^{j^{\prime} 1}\right)^{2} \alpha_{\perp}(i \omega)\right] \alpha_{c}(i \omega),
\end{aligned}
$$


where

$$
\mu_{n^{\prime} \ell^{\prime}, n \ell}=\frac{1}{\sqrt{3}} r_{n^{\prime} \ell^{\prime}, n \ell} C_{10 \ell 0}^{\ell^{\prime} 0}
$$

is the atomic transition dipole moment, $\Theta(x)$ is Heaviside's function, and $\Delta E_{n^{\prime} \ell^{\prime}, n \ell}^{0}=E_{n^{\prime} \ell^{\prime}}^{0}-E_{n \ell^{\prime}}^{0}$.

\section{LONG-RANGE POTENTIAL CURVES

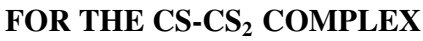

The atomic polarizability of $\operatorname{Cs}\left(6^{2} P\right)$ comes from the accurate calculations of transition dipole moments in Ref. [16], averaged over the $6 P_{1 / 2}$ and $6 P_{3 / 2}$ levels. The atomic transition energies are extracted from Ref. [17], and averaged over fine-structure levels. In Eq. (22), the summation is restricted to $n^{\prime}=7$ to 10 for $\ell^{\prime}=0$ and $n^{\prime}=5$ to 10 for $\ell^{\prime}=2$. We use a mixture of experimental and theoretical molecular data to compute the molecular polarizabilities at imaginary frequencies, which involve summation over all vibrational levels of all electronic states of $\mathrm{Cs}_{2}$. The $\mathrm{Cs}_{2}$ ground-state potential curve is taken from Ref. [18], and the one of the $B^{1} \Pi_{u}(6 s+6 p)$ state from Ref. [19]. The $A^{1} \Sigma_{u}^{+}(6 s+6 p)$ and $b^{3} \Pi_{u}(6 s+6 p)$ and their spin-orbit coupling are those fitted to reproduce the data of Ref. [20]. All the other electronic states come from quantum chemistry calculations performed in our group according to the method described in Ref. [21] as

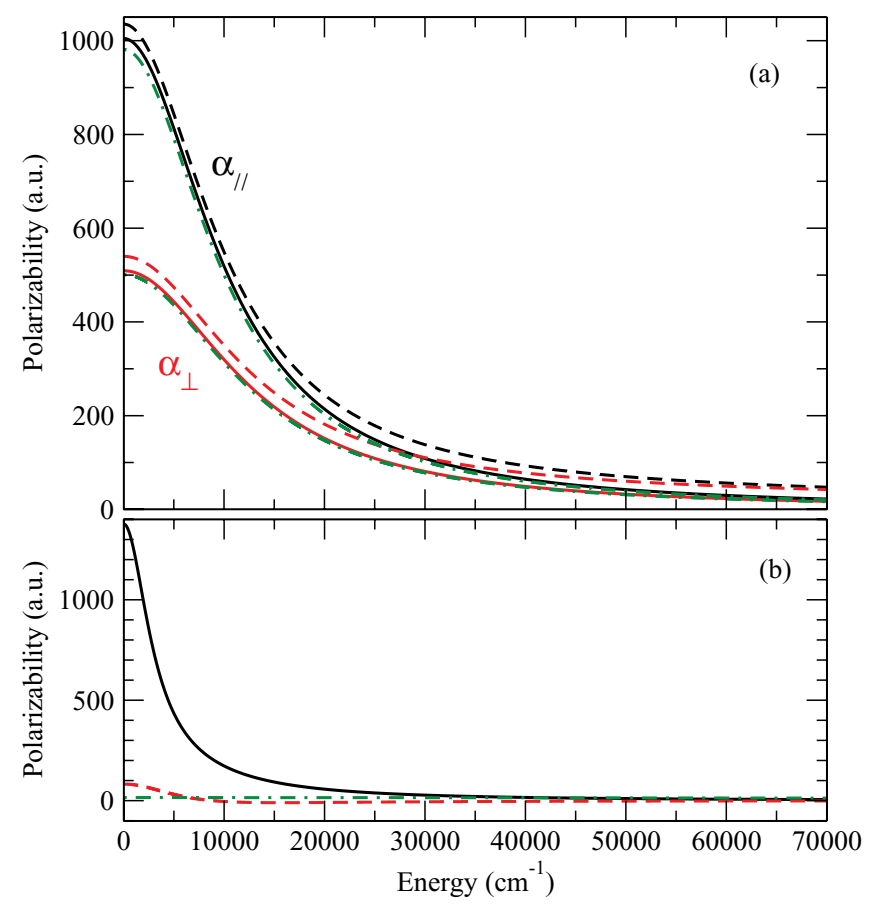

FIG. 2. (Color online) Calculated dipole polarizabilities in imaginary frequencies (a) for $\mathrm{Cs}_{2}\left(X^{1} \Sigma_{g}^{+}, v_{d}=0\right.$ ) (with the parallel and perpendicular components, full lines) and (b) for $\operatorname{Cs}\left(6^{2} P\right)$ (lower panel, full line). (a) Contribution of the sole $B^{1} \Pi_{u}(6 s+6 p)$ state to $\alpha_{\perp}$ and of the pair of $0_{u}^{+} A^{1} \Sigma_{u}^{+}(6 s+6 p)$ and $b^{3} \Pi_{u}(6 s+6 p)$ states to $\alpha_{\|}$(dot-dashed lines), and polarizability components including the core polarizability (dashed lines). (b) Magnitude of the second term of Eq. (12) (dashed line) and of the core polarizability (dot-dashed line). well as transition dipole moments, which will be presented in a separate publication. The dissociation energies of the atom-dimer complex are given by $\mathrm{Cs}_{2}$ rotational energies, $B_{0} j(j+1)$, where the rotational constant for the $v_{d}=0$ level of $\mathrm{Cs}_{2}$ is $B_{0}=1.17314 \times 10^{-2} \mathrm{~cm}^{-1}$ [18].

The variation of the dipole polarizability at imaginary frequencies is displayed in Fig. 2. The $B^{1} \Pi_{u}(6 s+6 p)$ electronic state contributes to the valence part for about $99 \%$ to $\alpha_{\perp}$, as well as the pair of states $A^{1} \Sigma_{u}^{+}(6 s+6 p)$ and $b^{3} \Pi_{u}(6 s+6 p)$ coupled by spin-orbit interaction to yield a pair of $0_{u}^{+}$states. The core polarizability represents only a few percentages of the total one, for the represented frequency domain. We numerically verified that the dissociation continua can be neglected.

The $C_{6}$ coefficients are first calculated for cesium in its ground-state $6^{2} S$ interacting with $\mathrm{Cs}_{2} X^{1} \Sigma_{g}^{+}\left(v_{d}=0, j\right)$ molecule, for which the quadrupole-quadrupole interaction is zero. The $\alpha_{\ell \ell^{\prime}}$ quantities now represent the actual dipole polarizability. Moreover there is no crossed term in the $C_{6}$ coefficients, and Eq. (33) reduces to

$$
\begin{aligned}
C_{6}= & -\frac{2}{\pi} \sum_{M} \sum_{j^{\prime} m_{j}^{\prime}} \frac{1}{[(1+M) !(1-M) !]^{2}} \\
& \times \frac{2 j+1}{2 j^{\prime}+1}\left(C_{1 M j m_{j}}^{j^{\prime} m_{j}^{\prime}}\right)^{2} \int_{0}^{+\infty} d \omega \alpha(i \omega) \\
& \times\left[\left(C_{10 j 0}^{j^{\prime} 0}\right)^{2} \alpha_{\|}(i \omega)+2\left(C_{11 j 0}^{j^{\prime} 1}\right)^{2} \alpha_{\perp}(i \omega)\right],
\end{aligned}
$$

where $\alpha(i \omega)$ is the atomic polarizability in the $6^{2} S$ state, including core contributions. It is worth noting that in the particular case $j=0$, Eq. (35) can be written in a similar form as for two $S$ atoms

$$
C_{6}=-\frac{3}{\pi} \int_{0}^{+\infty} d \omega \alpha(i \omega) \bar{\alpha}(i \omega),
$$

with $\bar{\alpha}=\left(\alpha_{\|}+2 \alpha_{\perp}\right) / 3$ the so-called isotropic polarizability of the dimer.

The results of our calculations are given in Table I and in Fig. 3. They are sorted by values of the projection $m_{J}$ of the total orbital momentum on the $Z$ axis, and for $m_{J}=0$, the parity \pm through the reflection symmetry with respect to any

TABLE I. The $C_{6}$ coefficients of the $\mathrm{Cs}_{2}\left(X^{1} \Sigma_{g}^{+}, v_{d}=0, j\right)+$ $\operatorname{Cs}\left(6^{2} S\right)$ long-range interaction calculated for $j=0$ to 4 . In analogy to a diatomic molecule, the $C_{6}$ are sorted by projections of the total orbital quantum number $m_{J}=m_{j}$ on the $Z$ axis (note that $\lambda=0$ ) and the parity \pm through the reflection symmetry with respect to a plane containing the axis. This yields the $\Sigma^{ \pm}, \Pi, \Delta, \Phi, \Gamma$ symmetries for $m_{J}=0, \pm 1, \pm 2, \pm 3, \pm 4$, respectively.

\begin{tabular}{lccccc}
\hline \hline Symmetry & $j$ & $C_{6}$ (a.u.) & Symmetry & $j$ & $C_{6}$ (a.u.) \\
\hline$\Sigma^{+}$ & 0 & -12101 & $\Pi$ & 4 & -12587 \\
& 1 & -12981 & $\Delta$ & 2 & -11473 \\
& 2 & -12729 & & 3 & -12101 \\
& 3 & -12688 & & 4 & -12330 \\
$\Pi$ & 4 & -12672 & $\Phi$ & 3 & -11369 \\
& 1 & -11662 & & 4 & -11902 \\
& 2 & -12415 & $\Gamma$ & 4 & -11302 \\
& 3 & -12541 & & & \\
\hline \hline
\end{tabular}




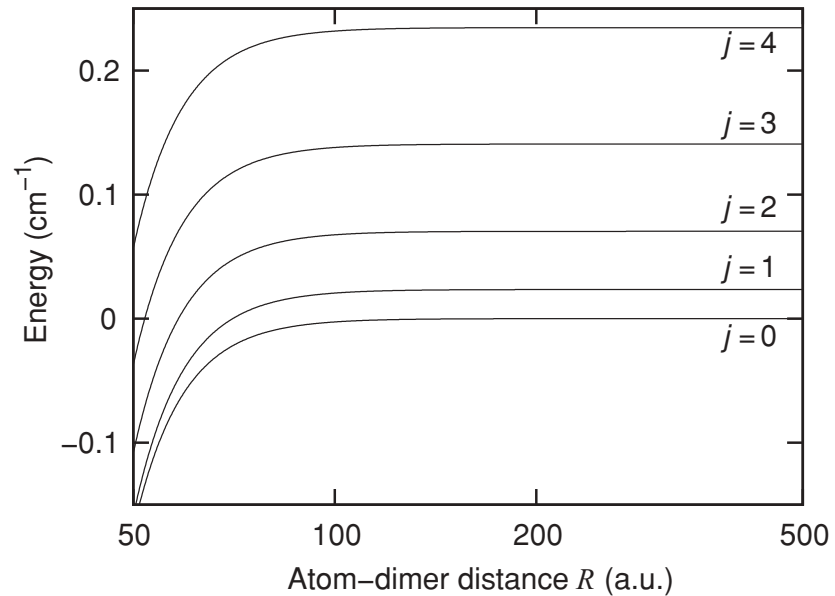

FIG. 3. Long-range potential energy curves $B_{0} j(j+1)+C_{6} / R^{6}$ of $\Sigma^{+}$symmetry $\left(m_{J}=0\right)$, as functions of the atom-dimer distance $R$ (in logarithmic scale), representing the interaction between $\operatorname{Cs}\left(6^{2} S\right)$ and $\mathrm{Cs}_{2}\left(X^{1} \Sigma_{g}^{+}, v_{d}=0, j\right)$ for $j=0$ to 4 . The related $C_{6}$ coefficients are given in Table I.

plane containing this axis. In analogy to diatomic molecules, these symmetries are labeled $\Sigma^{ \pm}, \Pi, \Delta, \Phi, \Gamma, \mathrm{H}$ for $m_{J}=0$, $\pm 1, \pm 2, \pm 3, \pm 4, \pm 5$, respectively. All the $C_{6}$ coefficients are negative, reflecting an attraction between the atom and the dimer, which is slightly less than twice the one for two interacting $\operatorname{Cs}\left(6^{2} S\right)$ atoms $(6840$ a.u. [6,22,23]). This can be
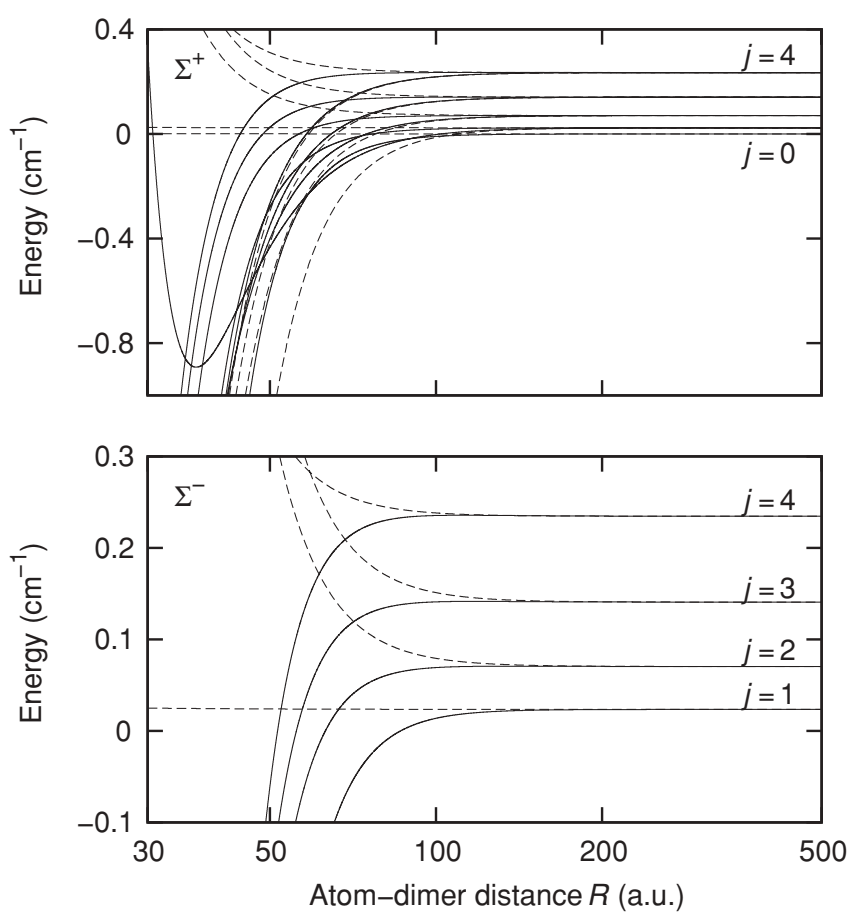

FIG. 4. Long-range potential energy curves $B_{0} j(j+1)+$ $C_{5} / R^{5}+C_{6} / R^{6}$ (full lines) and $B_{0} j(j+1)+C_{5} / R^{5}$ (dashed lines) as functions of the atom-dimer distance $R$ (in logarithmic scale), for the $\Sigma^{+}$and $\Sigma^{-}$symmetries $\left(m_{J}=0\right)$, representing the interaction between $\operatorname{Cs}\left(6^{2} P\right)$ and $\operatorname{Cs}_{2}\left(X^{1} \Sigma_{g}^{+}, v_{d}=0, j\right)$ for $j=0$ to 4 . The related $C_{5}$ and $C_{6}$ coefficients are given in Table II.
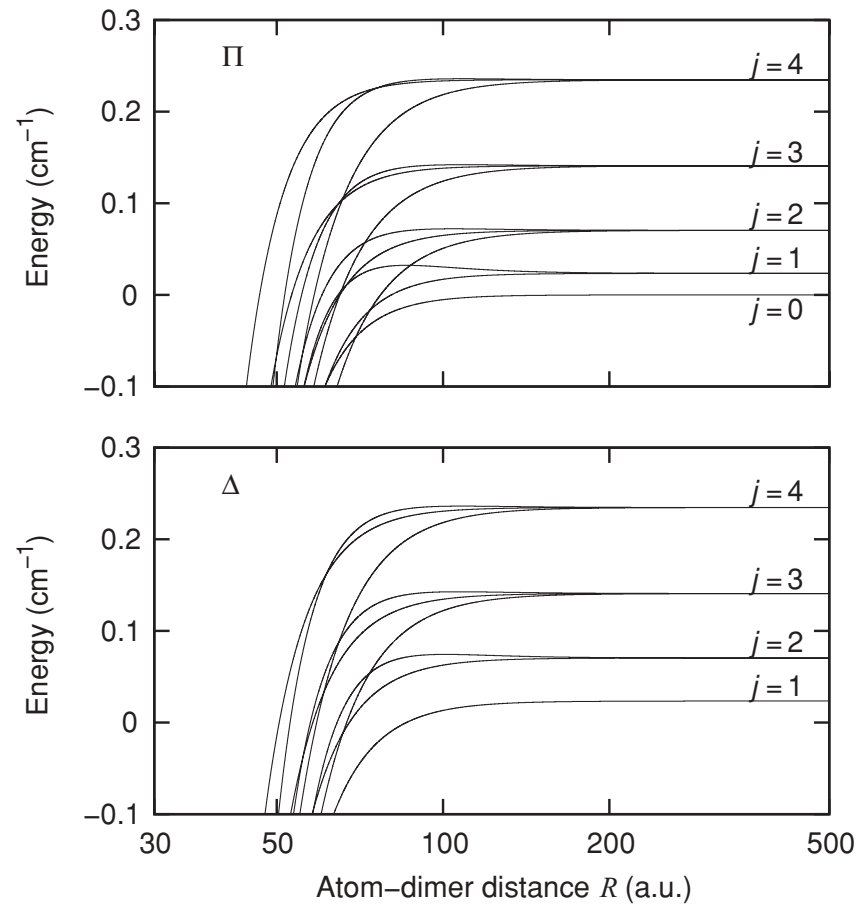

FIG. 5. Same as Fig. 4 for curves of $\Pi\left(m_{J}=1\right)$ and $\Delta\left(m_{J}=2\right)$ symmetries.

understood from the simple form of Eq. (36), considering that the static dipole polarizabilities of a ground-state $\mathrm{Cs}$ atom and of a ground-state $\mathrm{Cs}_{2}$ molecule are, respectively, $\approx 402$ a.u. [22] and $\approx 707$ a.u. [24,25]. In addition, no curve crossing are visible on Fig. 3 as all $C_{6}$ coefficients have close values. Therefore, the validity of the long-range expansion is limited at short distances by the overlap of the electronic clouds. With $\left\langle r_{6 S}^{2}\right\rangle=42$ a.u. [26], we estimate the value of the LeRoy radius to $R_{L R} \sim 40-45$ a.u.

The potential energy curves for the long-range interaction between $\mathrm{Cs}\left(6^{2} P\right)$ and $\mathrm{Cs}_{2}\left(X^{1} \Sigma_{g}^{+}, v_{d}=0, j\right)$ are displayed in Figs. 4, 5, and 6. For the $\Sigma$ symmetries (see Fig. 4), the curves calculated in paper I (containing only the $C_{5}$ term) are also shown. The related $C_{5}$ (from paper I) and $C_{6}$ coefficients are listed in Table II.

The number of states appearing in a given symmetry depends on the number of allowed values of the $m_{j}$ and $\lambda$ quantum numbers. For example, in the $\Sigma^{ \pm}, j=1$ symmetry, three couples $\left(m_{j}, \lambda\right)$ are allowed: $(-1,1),(0,0)$, and $(1,-1)$. Two eigenvectors are symmetric combinations of $(-1,1)$ and $(1,-1)$ and/or contain a $(0,0)$ contribution: They are labeled $\Sigma^{+}$. One eigenvector is an antisymmetric combination of $(-1,1)$ and $(1,-1)$ : It is labeled $\Sigma^{-}$(see Table I of paper I).

Most potential curves are attractive, even if they are characterized by either a positive $C_{5}$ coefficient or a positive $C_{6}$ coefficient. A positive $C_{5}$ coefficient and a negative $C_{6}$ coefficient would, in principle, give birth to a long-range potential barrier. But in general, the absolute value of the $C_{5}$ coefficient is so small that the barrier is not visible on the scale of the graph, which happens, for example, for all $\Sigma^{-}$states. However, a few tiny potential barriers are visible. For instance, 

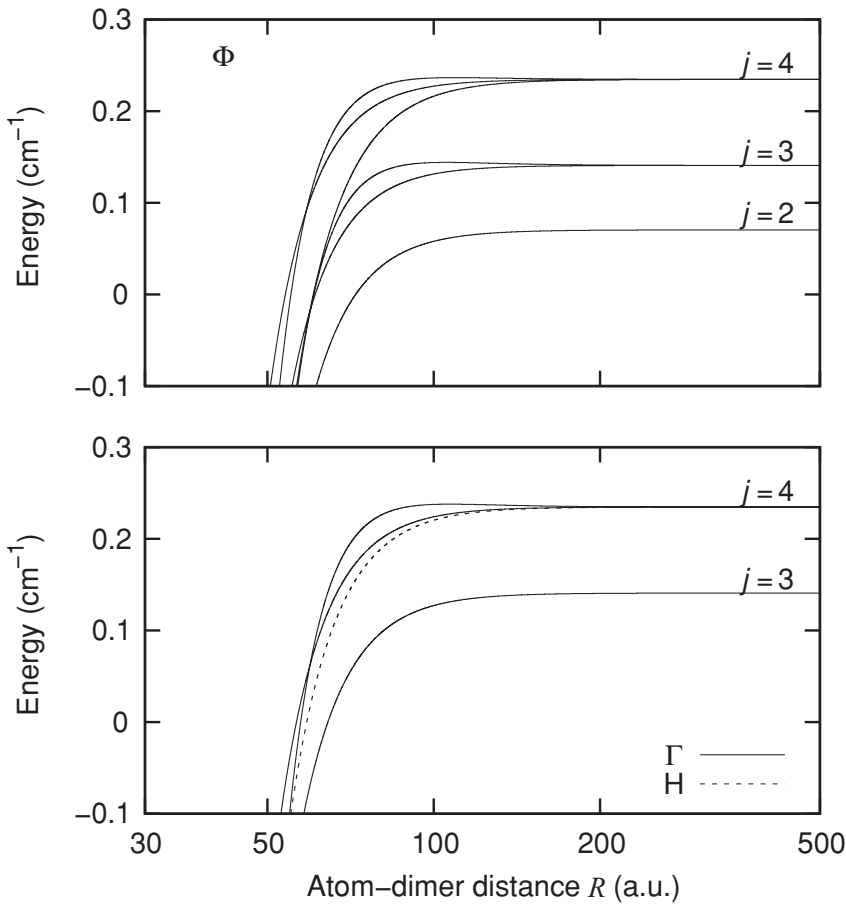

FIG. 6. Same as Fig. 5 for curves of $\Phi\left(m_{J}=3\right)$ symmetry (upper panel), and of $\Gamma\left(m_{J}=4\right)$ (solid line) and $\mathrm{H}\left(m_{J}=5\right)$ (dashed line) symmetries (lower panel).

the highest one is found for $\Pi$ symmetry with a height of about $0.01 \mathrm{~cm}^{-1}$ in the curve correlated to the $j=1$ rotational state.
When combined with a negative $C_{5}$ coefficient, a positive $C_{6}$ coefficient would in principle induce a long-range well. However, in most cases, the imbalance between both contributions is such that the minimum of the well is located deeply inside the region of electronic exchange. A remarkable exception is the $\Sigma^{+}$state with $j=1$, for which $C_{5}=-1674$ a.u. and $C_{6}=51249$ a.u. (see Fig. 4). This well is $0.9 \mathrm{~cm}^{-1}$ deep, and its minimum is located at $R=37$ a.u., slightly below the LeRoy radius ( $\sim 45$ a.u.).We can expect that this long-range well will indeed exist, but it will certainly be modified by rotational and nonadiabatic couplings discussed above and by the electronic exchange. Such a well will carry bound levels of $\mathrm{Cs}_{3}$, whose lifetime could be affected by predissociation effects related to neighboring potential curves. Therefore, the precise characterization of this well and of its bound levels requires quantum-chemical calculations.

In paper I, we found that the low- $R$ limit of our perturbation analysis $R_{m}=102$ a.u. was imposed by the crossings between curves with different values of $j$. We see in Fig. 4 that the typical position of these crossings is only slightly modified by the $C_{6}$ contribution. However, it is worth noting that the crossing range coincides with the one in which the secondorder contribution competes with the first-order contribution. As a consequence, the nonadiabatic couplings emerging in the crossing region, mentioned in paper I, should include both first-order and second-order terms. The resulting couplings will mix states characterized by a given symmetry $\left(\Sigma^{ \pm}, \Pi, \ldots\right)$ and a given $j$, with states characterized by the same symmetry and by $j^{\prime}=j \pm 2$.

TABLE II. The $C_{5}$ and $C_{6}$ coefficients of the $\mathrm{Cs}_{2}\left(X^{1} \Sigma_{g}^{+}, v_{d}=0, j\right)+\mathrm{Cs}\left(6^{2} P\right)$ long-range interaction calculated for $j=0$ to 4 . In analogy to a diatomic molecule, the states are sorted by projections of the total orbital quantum number $m_{J}=m_{j}+\lambda$ on the $Z$ axis and by the parity \pm after the reflection symmetry through any plane containing this axis. The values for $C_{5}$ are taken from paper I.

\begin{tabular}{|c|c|c|c|c|c|c|c|}
\hline Symmetry & $j$ & $C_{5}$ (a.u.) & $C_{6}$ (a.u.) & Symmetry & $j$ & $C_{5}$ (a.u.) & $C_{6}$ (a.u.) \\
\hline \multirow[t]{9}{*}{$\Sigma^{+}$} & 0 & 0 & -42704 & $\Pi$ & 4 & -739 & 671 \\
\hline & 1 & -1674 & 51249 & & 4 & 108 & -15884 \\
\hline & 1 & 0 & -21562 & & 4 & 522 & -47279 \\
\hline & 2 & -913 & 12128 & $\Delta$ & 1 & -279 & -18694 \\
\hline & 2 & 116 & -16885 & & 2 & -140 & -21244 \\
\hline & 3 & -796 & 4923 & & 2 & 1136 & -95614 \\
\hline & 3 & 145 & -15420 & & 3 & -835 & -1624 \\
\hline & 4 & -755 & 2251 & & 3 & -87 & -19563 \\
\hline & 4 & 157 & -14835 & & 3 & 736 & -65454 \\
\hline \multirow[t]{4}{*}{$\Sigma^{-}$} & 1 & 0 & -43920 & & 4 & -721 & -2643 \\
\hline & 2 & 399 & -45131 & & 4 & -11 & -17153 \\
\hline & 3 & 465 & -45333 & & 4 & 623 & -56200 \\
\hline & 4 & 489 & -45407 & $\Phi$ & 2 & -399 & -16589 \\
\hline \multirow[t]{9}{*}{$\Pi$} & 0 & 0 & -23605 & & 3 & -245 & -18030 \\
\hline & 1 & 0 & -29303 & & 3 & 1175 & -103161 \\
\hline & 1 & 1116 & -79756 & & 4 & -783 & -5057 \\
\hline & 2 & -964 & 7305 & & 4 & -161 & -17444 \\
\hline & 2 & -19 & -22961 & & 4 & 835 & -76003 \\
\hline & 2 & 584 & -50736 & $\Gamma$ & 3 & -465 & -15420 \\
\hline & 3 & -783 & 2496 & & 4 & -320 & -16392 \\
\hline & 3 & 64 & -17295 & & 4 & 1208 & -107555 \\
\hline & 3 & 532 & -48103 & $\mathrm{H}$ & 4 & -507 & -14676 \\
\hline
\end{tabular}




\section{CONCLUSIONS}

Using a second-order perturbation approach, we have shown that the long-range interaction between an excited $\mathrm{Cs}\left(6^{2} P\right)$ atom and a $\mathrm{Cs}_{2}\left(X^{1} \Sigma_{g}^{+}, v_{d}=0, j\right)$ molecule is significantly modified compared to the first-order quadrupolequadrupole interaction. Most potential curves exhibit an attractive behavior, except in a few cases where either a low potential barrier or a long-range well is visible. The barriers could prevent the collision from occurring in the ultracold domain. The long-range well could accommodate bound levels of the excited cesium trimer, just like in the well-known long-range wells in alkali-metal dimers [27]. However, the inclusion of the rotation of the trimer and of other interactions such as spin-orbit will most probably limit the lifetime of such levels, which could be much shorter than the natural lifetime. Furthermore one can speculate that the richness of these potential curves may well induce double-well structures such as the one observed in $\mathrm{Cs}_{2}$ [28] and $\mathrm{Rb}_{2}$ [29], which would favor the formation of ultracold stable cesium trimers by spontaneous emission.

The validity of the present approach is limited to a range of distances well beyond the radius defined by the conventional LeRoy criterion, due to the low-energy spacings between molecular rotational levels. This is actually the main difference compared to atom-atom long-range interaction, which completely changes the physical conditions for PA of an atom with a molecule. The next step in the theory is to include nonadiabatic couplings between curves related to different rotational levels, which is currently in progress in our group. They will induce avoided crossings that may generate a complex collisional dynamics.
While the present theory is general, it is difficult to predict if this situation is similar for all alkali-metal systems, either homonuclear or heteronuclear, as it strongly depends on the balance between the influence of the various parameters, namely, the quadrupole moment of the atom and the dimer and their dipole polarizability. As already stressed in paper I, other effects should be taken in account, such as atomic spin-orbit interaction. The fine-structure splitting in cesium $\left(554.1 \mathrm{~cm}^{-1}\right)$ is much larger than the rotational energy of $\mathrm{Cs}_{2}$, so that this effect will not dramatically change our description. However, it is important to stress that the above treatment was developed on the basis of the Russel-Sanders (or LS) coupling case.

The inclusion of the hyperfine structure (a few hundreds of $\mathrm{MHz}$ for the $\mathrm{Cs}\left(6 P_{1 / 2,3 / 2}\right)$ atom, and a few tens of $\mathrm{kHz}$ for the $\mathrm{Cs}_{2}$ ground-state molecule [30]) will add to the complexity of the potential curves of Figs. 4, 5, and 6, while probably having a limited influence on the overall dynamics. It will not modify the main conclusion of the present study: The photoassociation of a ground-state $X^{1} \Sigma_{g}^{+}$alkali-metal dimer molecule with a ground-state $n^{2} S_{1 / 2}$ alkali-metal atom is generally possible by exciting the dimer-atom system with a laser field red-detuned from the $n^{2} S_{1 / 2} \rightarrow n^{2} P_{1 / 2,3 / 2}$ atomic transition.

\section{ACKNOWLEDGMENTS}

We thank M. Aymar for kindly providing us with $\mathrm{Cs}_{2}$ potential curves and transition dipole moments. This work was done with the support of Triangle de la Physique under contract 2008-007T-QCCM (Quantum Control of Cold Molecules) and the National Science Foundation under grant PHY-0855622.
[1] M. Lepers, O. Dulieu, and V. Kokoouline, Phys. Rev. A 82, 042711 (2010).

[2] L. D. Carr and J. Ye, New J. Phys. 11, 055009 (2009).

[3] O. Dulieu and C. Gabbanini, Rep. Prog. Phys. 72, 086401 (2009).

[4] C. Chin, R. Grimm, P. Julienne, and E. Tiesinsga, Rev. Mod. Phys. 82, 1225 (2010).

[5] J. Mitroy and M. W. J. Bromley, Phys. Rev. A 68, 052714 (2003).

[6] A. Derevianko, S. G. Porsev, and J. F. Babb, At. Data Nucl. Data Tables 96, 323 (2010).

[7] N. Bouloufa, A. Crubellier, and O. Dulieu, Phys. Scripta T113, 014014 (2009).

[8] M. Marinescu and A. F. Starace, Phys. Rev. A 55, 2067 (1997).

[9] B. Bussery-Honvault, F. Dayou, and A. Zanchet, J. Chem. Phys. 129, 234302 (2008).

[10] B. Bussery-Honvault and F. Dayou, J. Phys. Chem. A 113, 14961 (2009).

[11] S. Kotochigova, New J. Phys 12, 073041 (2010).

[12] R. J. Le Roy, Can. J. Phys. 52, 246 (1974).

[13] D. Spelsberg, T. Lorenz, and W. Meyer, J. Chem. Phys. 99, 7845 (1993).
[14] C. Zhu, A. Dalgarno, S. G. Porsev, and A. Derevianko, Phys. Rev. A 70, 032722 (2004).

[15] D. A. Varshalovich, A. N. Moskalev, and V. K. Khersonskii, Quantum Theory of Angular Momentum (World Scientific Publishing Company, Leningrad, 1988).

[16] U. S. E. Iskrenova-Tchoukova and M. S. Safronova, J. Comput. Meth. Sci. Eng. 7, 521 (2007).

[17] C. Moore, Atomic Energy Levels, Vol. 2 (Chromium through Niobium) (U.S. Government Printing Office, Washington, DC, 1952).

[18] C. Amiot, O. Dulieu, R. F. Gutterres, and F. Masnou-Seeuws, Phys. Rev. A 66, 052506 (2002).

[19] U. Diemer, R. Duchowicz, M. Ertel, E. Mehdizadeh, and W. Demtröder, Chem. Phys. Lett. 164, 419 (1989).

[20] J. G. Danzl, E. Haller, M. Gustavsson, M. J. Mark, R. Hart, N. Bouloufa, O. Dulieu, H. Ritsch, and H.-C. Nägerl, Science 321, 1062 (2008).

[21] M. Aymar and O. Dulieu, J. Chem. Phys. 122, 204302 (2005).

[22] A. Derevianko, W. R. Johnson, M. S. Safronova, and J. F. Babb, Phys. Rev. Lett. 82, 3589 (1999).

[23] A. Derevianko, J. F. Babb, and A. Dalgarno, Phys. Rev. A 63, 052704 (2001). 
[24] J. Deiglmayr, M. Aymar, R. Wester, M. Weidemüller, and O. Dulieu, J. Chem. Phys. 129, 064309 (2008).

[25] The quantity $2 \alpha_{c}\left(\mathrm{Cs}^{+}\right)=30.8$ a.u. deduced from Ref. [22] has been added to the one of Ref. [24].

[26] M. Aymar (private communication).

[27] W. C. Stwalley, Y. H. Uang, and G. Pichler, Phys. Rev. Lett. 41, 1164 (1978).
[28] A. Fioretti, D. Comparat, A. Crubellier, O. Dulieu, F. Masnou-Seeuws, and P. Pillet, Phys. Rev. Lett. 80, 4402 (1998).

[29] C. Gabbanini, A. Fioretti, A. Lucchesini, S. Gozzini, and M. Mazzoni, Phys. Rev. Lett. 84, 2814 (2000).

[30] J. Aldegunde and J. M. Hutson, Phys. Rev. A 79, 13401 (2009). 